\title{
ARTICLE \\ EPHA2 feedback activation limits the response to PDE $\delta$ inhibition in KRAS-dependent cancer cells
}

\author{
Yue-hong Chen ${ }^{1,2}$, Hao Lv ${ }^{1,3}$, Ning Shen ${ }^{1}$, Xiao-min Wang ${ }^{1,3}$, Shuai Tang ${ }^{1}$, Bing Xiong ${ }^{4}$, Jian Ding ${ }^{1,3}$, Mei-yu Geng ${ }^{1,3}$ and Min Huang ${ }^{1,3}$
}

\begin{abstract}
KRAS is one of the most important proto-oncogenes. Its mutations occur in almost all tumor types, and KRAS mutant cancer is still lack of effective therapy. Prenyl-binding protein phosphodiesterase- $\delta$ (PDE $\delta$ ) is required for the plasma membrane association and subsequent activation of KRAS oncogenic signaling. Recently, targeting PDE $\delta$ has provided new promise for KRAS mutant tumors. However, the therapeutic potential of PDE $\delta$ inhibition remains obscure. In this study, we explored how PDE $\delta$ inhibition was responded in KRAS mutant cancer cells, and identified KRAS mutant subset responsive to PDE $\delta$ inhibition. We first performed siRNA screen of KRAS growth dependency of a small panel of human cancer lines, and identified a subset of KRAS mutant cancer cells that were highly dependent on KRAS signaling. Among these cells, only a fraction of KRAS-dependent cells responded to PDE $\delta$ depletion, though KRAS plasma membrane association was effectively impaired. We revealed that the persistent RAF/MEK/ERK signaling seemed responsible for the lack of response to PDE $\delta$ depletion. A kinase array further identified that the feedback activation of EPH receptor A2 (EPHA2) accounted for the compensatory activation of RAF/MEK/ERK signaling in these cells. Simultaneous inhibition of EPHA2 and PDE $\delta$ led to the growth inhibition of KRAS mutant cancer cells. Together, this study gains a better understanding of PDE $\delta$-targeted therapeutic strategy and suggests the combined inhibition of EPHA2 and PDE $\delta$ as a potential therapy for KRAS mutant cancer.
\end{abstract}

Keywords: KRAS; anticancer therapy; PDES; EPHA2; RAF/MEK/ERK signaling

Acta Pharmacologica Sinica (2020) 41:270-277; https://doi.org/10.1038/s41401-019-0268-y

\section{INTRODUCTION}

RAS proteins, encoded by an important family of protooncogenes, belong to a class of proteins called small GTPases and are involved in transmitting signals within cells [1, 2]. Alterations to RAS proto-oncogenes, in the form of point mutations, have been identified in almost all tumors. Oncogenic mutations to specific amino acids maintain RAS in a constitutively active GTP-bound state, resulting in the continuous activation of the RAS signal transduction pathway [3-5]. This signal is transmitted to downstream pathways, particularly the RAS/RAF/ MEK/ERK and PI3K/AKT/mTOR signaling pathways, which further affect a plethora of cellular events, including the cell cycle progression, cell transformation, proliferation, apoptosis, migration, and differentiation $[3,6,7]$. The most commonly mutated RAS isoform is KRAS4B, often referred to as KRAS in human cells, which occurs in more than $90 \%$ of pancreatic tumors, $45 \%$ of colorectal tumors and $30 \%$ of lung tumors; these facts make the KRAS protein an attractive target in anticancer drug discovery [8]. However, decade-long efforts in exploiting this target have failed to yield clinically effective therapies, which is believed to be due to the special structure of the KRAS protein, the high affinity of KRAS for GTP and the complexity of the downstream signaling pathways of KRAS [2, 8-10].
Notably, recent studies have highlighted a new therapeutic strategy that targets a key step in the process of KRAS membrane localization [1]. The KRAS protein requires localization to the inner leaflet of the plasma membrane for oncogenic activity. The guanine nucleotide dissociation inhibitor (GDI)-like solubilization factor phosphodiesterase- $\delta$ (PDE $\delta$ ) is required for the correct localization and signaling of farnesylated KRAS [11]. PDE $\delta$ belongs to a large family of phosphodiesterases (PDEs). It possesses a hydrophobic pocket and could interact with a farnesylated hydrophobic cysteine residue at the C-terminus of the KRAS protein [12]. This interaction sequesters KRAS from the cytosol, preventing KRAS from binding to endomembranes and thereby facilitating its diffusion in the cytoplasm. Interfering with the binding of mammalian PDE $\delta$ to KRAS has been shown to provide a novel way to suppress oncogenic RAS signaling. Zimmermann et al. discovered the first small molecule inhibitor, deltarasin, that bound the farnesyl-binding pocket of PDE $\delta$ and disrupted KRAS signaling [13].

While this novel strategy appears to be promising for conquering this long-pursued target, it is also noted that in addition to KRAS, PDE $\delta$ is crucial for maintaining the membrane localization of other RAS family members, such as palmitoylated $\mathrm{H}$ - and N-RAS and solely farnesylated RHEB [12]. Moreover, PDE $\delta$ is reported to have an interactome composed of multiple proteins,

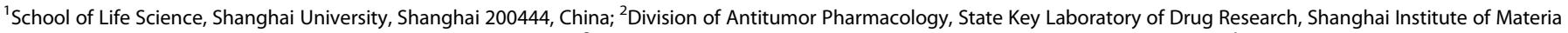

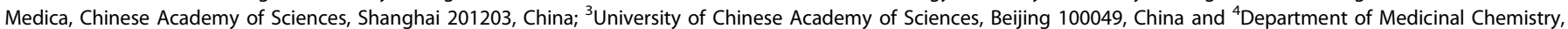
Shanghai Institute of Materia Medica, Chinese Academy of Sciences, Shanghai 201203, China

Correspondence: Min Huang (mhuang@simm.ac.cn)
}

Received: 23 April 2019 Accepted: 3 June 2019

Published online: 17 July 2019 
such as RAB23, CDC42, and CNP proteins [14]. Inhibition of PDE $\delta$ is expected to have broad cellular effects, yielding uncertainties in KRAS-dependent cancer cells. Moreover, the anticancer activity of newly discovered small molecule inhibitors targeting PDE $\delta$ remains obscure. The mechanism of deltarasin, the first reported KRAS-PDE $\delta$ inhibitor, appears not to be limited to KRAS signaling [13]. The complexity of the role of PDE $\delta$ and the mechanism of action of PDE $\delta$ inhibition in KRAS-dependent cells require an indepth understanding of PDE $\delta$-targeted therapies.

This study aims to gain a better understanding of the effects of PDES inhibition in KRAS mutant cancer cells. Given the heterogeneous proprieties of KRAS mutant cancer, this study also seeks to identify the tumor subset responsive to PDE $\delta$ inhibition. By profiling a panel of KRAS mutant cancer cell lines, we discovered that PDE $\delta$ inhibition was effective in a small proportion of KRAS mutant cancer and was closely associated with the alteration of RAF/MEK/ERK signaling in these cells. We also discovered that the feedback activation of EPH receptor A2 (EPHA2) accounted for the compensatory activation of RAF/MEK/ERK signaling, leading to resistance to PDE $\delta$ inhibition. We therefore propose a new therapeutic strategy that concurrently inhibits PDE $\delta$ and EPHA2 for the treatment of KRAS mutant cancer.

\section{MATERIALS AND METHODS}

Reagents and antibodies

Deltarasin (S7224), BVD-523 (S7854), AZD6244 (S1008), and BMS754807 (S1124) were obtained from Selleck (Houston, TX, USA). ALWII-41-27 was obtained from AbMole (Houston, TX, USA). The antibodies used in this study were as follows: antibodies against phospho-c-RAF (Ser338) (9427s), c-RAF (53745s), phospho-p44/42 MAPK (ERK1/2) (Thr202/Tyr204) (4370L), p44/42 MAPK (ERK1/2) (4695s), phospho-AKT (Ser473) (4060s), AKT (4691s), phospho-p70 S6 kinase (Thr389) (9234s), p70 S6 kinase (2708s), phospho-EPHA2 (Tyr594) (3970s), phospho-EphA2 (Tyr588) (12677s), EPHA2 (6997s), IGF-I receptor $\beta$ (3027s), phospho-IGF-I receptor $\beta$ (Tyr1135) (3918s), phospho-WNK-1 (Thr60) (4946s), and phospho-GSK-3 $\beta$ (Ser9) (5558s) were all from Cell Signaling Technology (Cambridge, MA, USA). An antibody against KRAS (101667-T32) was purchased from Sino Biological, and an antibody against PDE6D (ab96825) was purchased from Abcam (Shanghai, China). GAPDH (60004-1-lg) was obtained from Proteintech (Rosemont, IL, USA).

\section{Cell lines}

$\mathrm{NCl}-\mathrm{A} 549, \mathrm{NCl}-\mathrm{H} 23, \mathrm{NCl}-\mathrm{H} 1792, \mathrm{NCl}-\mathrm{H} 441, \mathrm{H} 460$, and $\mathrm{NCl}-\mathrm{H} 358$ cells were obtained from the American Type Culture Collection (ATCC, Manassas, VA, USA). NCl-SW1573, NCl-H2030, AsPC-1, Panc 02.03, Panc 10.05, and H1944 cells were obtained from Cobioer (Nanjing, China). All cell lines were routinely authenticated by short tandem repeat analysis (Genesky Biotechnologies, Shanghai, China) and were maintained in the appropriate culture medium suggested by the suppliers.

Plasmid, shRNA, and siRNA transfection. A KRAS-GFP plasmid (HG12259-ANG) was obtained from Sino Biological (Beijing, China). For siRNA transfection, cells were plated at 30\%-60\% confluence in the OPTI-MEM serum-free medium and transfected with a specific siRNA duplex using Lipofectamine RNAiMAX (Invitrogen, (A, USA), according to the manufacturer's instructions. siRNAs were ordered from Shanghai GenePharma (Shanghai, China). The sequences of the scrambled negative control (NC) and siRNAs targeting the indicated genes were as follows:

NC 5'-UUCUCCGAACGUGUCACGUTT-3'

KRAS \#1 5'-GAGGAGUACAGUGCAAUGATT-3'

KRAS \#2 5'-GGAUUCCUACAGGAAGCAATT-3'

PDE6D \#1 5'-CUUCGACAGAACAAAUGGATT-3'

PDE6D \#2 5'-AUGCCUAGAAGAAUGGUUCUTT-3'
SIEPHA2 \#1 5'-GCUCAAGUUUACUGUACGUTT-3' SIEPHA2 \#2 5'-GUCCGUGUCUACUACAAGATT-3'

Cell viability assay

SRB assay. Cells were seeded in 96-well plates overnight. Sulforhodamine B assays (Thermo Fisher Scientific) were performed after treatment with the indicated reagents for $72 \mathrm{~h}$. The absorbance (optical density $[O D]$ ) was read at a wavelength of $515-560 \mathrm{~nm}$ on an ELISA plate reader. The growth inhibition rate was calculated according to $(O D$ treated $/ O D$ control) $\times 100 \%$, in which untreated cells served as the control. The experiment was repeated for at least three times. The data represent the means of triplicate analyses of a representative experiment.

Crystal violet assay. Cells were seeded in 12-well plates overnight. A crystal violet assay was performed after treatment with the indicated reagents for $96 \mathrm{~h}$. $O D$ values were read at a wavelength of 515-560 nm on an ELISA plate reader. The growth inhibition rate was calculated according to $(O D$ treated $/ O D$ control) $\times 100 \%$, in which untreated cells served as the control. The experiment was repeated for at least three times. The data represent the mean from four replicate analyses of a representative experiment.

Immunoblotting analysis

Cells were lysed using preheated $2 \%$ SDS by vortexing vigorously for $10-15 \mathrm{~s}$ at maximum speed, followed by boiling for $30 \mathrm{~min}$. The same amount of protein was subjected to SDS-PAGE, transferred to nitrocellulose membranes, and blocked for $1 \mathrm{~h}$ at room temperature with $3 \% \mathrm{BSA}$ in $1 \times$ Tris-buffered saline-Tween-20 (TBST; $25 \mathrm{mM}$ Tris, $150 \mathrm{mM} \mathrm{NaCl}$, and $2 \mathrm{mM} \mathrm{KCl}, \mathrm{pH} 7.4$, supplemented with $0.2 \%$ Tween-20). Blotting was performed with primary antibodies at $4{ }^{\circ} \mathrm{C}$ overnight. After washing the membranes with TBST three times for a total of $30 \mathrm{~min}$, horseradish peroxidase-conjugated secondary antibodies were incubated at room temperature for $1 \mathrm{~h}$. The membranes were washed three times with TBST for $30 \mathrm{~min}$, and the proteins were visualized with an enhanced chemiluminescence assay (Thermo Fisher Scientific) or Femto chemiluminescence assay (Thermo Fisher Scientific).

Fluorescence microscopy

Cells were fixed with $4 \%$ paraformaldehyde and then treated with DAPI for 15-20 min. Images were captured using a laser scanning confocal microscope (Lavision Biotec TriM Scope). At least 50 cells were counted per sample for quantification. Cells with apparent KRAS plasma membrane localization were counted and then normalized to the total cell counts. The data represent the means of three independent experiments.

Phospho-kinase antibody arrays and phospho-RTK array kit Cells $\left(1 \times 10^{6}\right)$ seeded in a $10-\mathrm{cm}$ dish were treated with the indicated reagents for $48 \mathrm{~h}$. Cells were lysed, and phosphorylation changes were detected by a Phospho-RTK Array Kit (\#ARY001B, $R \& D$ Systems) and phospho-kinase antibody array (ARY003B, R\&D Systems) following the manufacturer's instructions.

\section{RESULTS}

KRAS dependency varies in KRAS mutant cancer cell lines Previous studies have revealed that KRAS mutations, though they occur widely in human cancer, may not necessarily give rise to KRASdependent cell growth [15]. As such, we first screened the growth dependency of a small panel of human cancer cell lines bearing welldocumented KRAS-activating mutations, mainly highly frequent mutations to G12 residuals (Fig. 1a). Indeed, knocking down KRAS expression using two independent siRNAs resulted in diverse effects on cell growth in these cells. Among the 12 tested cell lines, four cell lines, namely, Panc 02.03, H460, H1792, and H358, showed substantial growth suppression. Some cell lines, such as SW1573, H23, A549, 
a

\begin{tabular}{|c|c|c|}
\hline Cell Lines & $\begin{array}{c}\text { Cancer } \\
\text { Types }\end{array}$ & $\begin{array}{c}\text { Kras } \\
\text { Mutation }\end{array}$ \\
\hline SW1573 & NSCLC & G12C \\
\hline A549 & NSCLC & G12S \\
\hline H460 & NSCLC & Q61H \\
\hline H358 & NSCLC & G12C \\
\hline H23 & NSCLC & G12C \\
\hline H1792 & NSCLC & G12C \\
\hline H2030 & NSCLC & G12C \\
\hline H441 & NSCLC & G12V \\
\hline H1944 & NSCLC & G13D \\
\hline AsPC-1 & PDAC & G12D \\
\hline Panc 02.03 & PDAC & G12D \\
\hline Panc 10.05 & PDAC & G12D \\
\hline
\end{tabular}

b

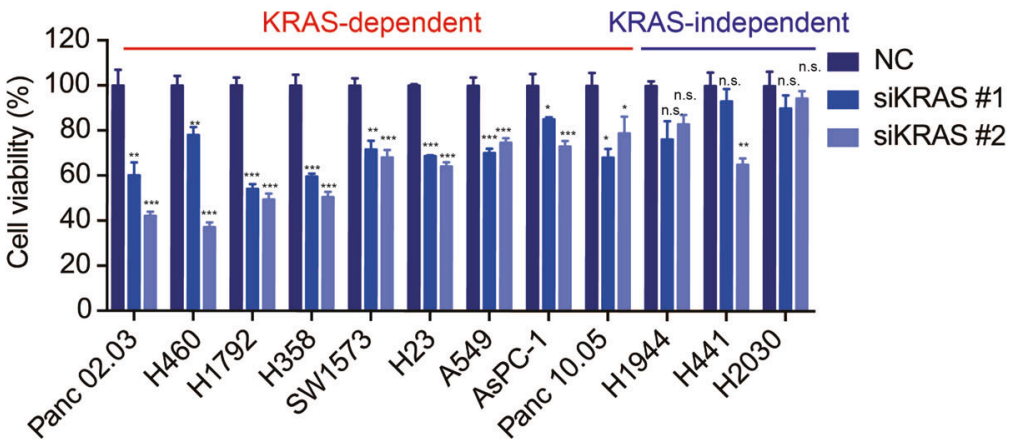

Panc

$02.03 \quad \underline{\mathrm{H}} 460 \quad \underline{\mathrm{H} 1792} \quad \underline{\mathrm{H} 358} \mathrm{SW} 1573 \quad \mathrm{H} 23$

SiKRAS NC\#1 \#2 NC\#1 \#2 NC\#1 \#2 NC\#1 \#2 NC\#1 \#2 NC\#1\#2

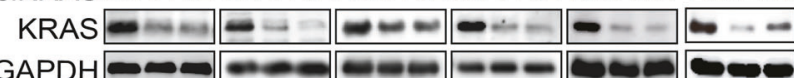

Panc

A549 AsPC-1 $10.05 \quad \mathrm{H} 1944 \quad \mathrm{H} 441 \quad \mathrm{H} 2030$

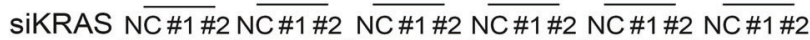

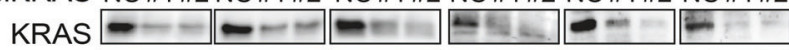
GAPDH
C

d

e

\section{$\mathrm{H} 460 \quad \mathrm{H} 358 \quad \mathrm{SW} 1573$}

siKRAS NC \#1 \#2 NC \#1 \#2 NC \#1 \#2

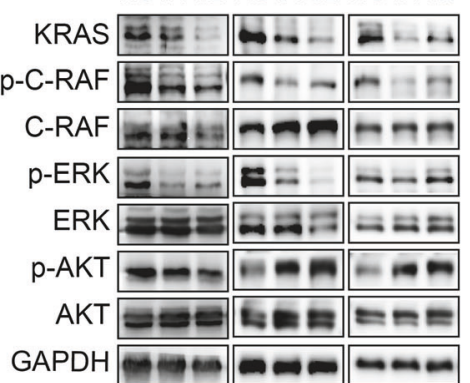

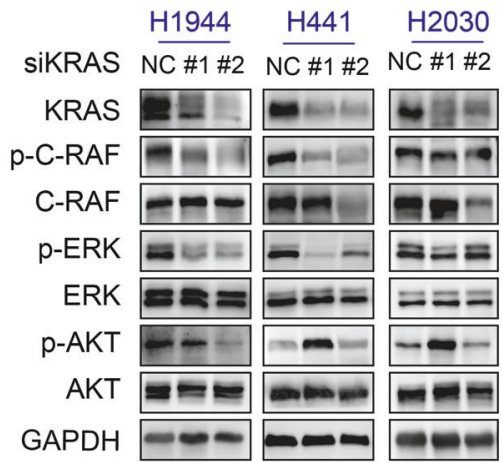

SIKRAS $\overline{\mathrm{NC \# 1} \# 2} \overline{\mathrm{NC \# 1 \# 2}} \overline{\mathrm{NC \# 1 \# 2}}$

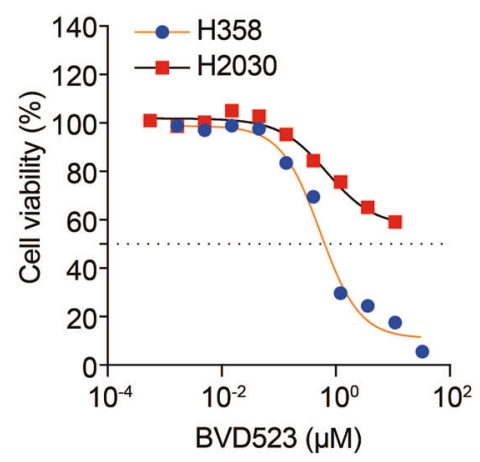

Fig. 1 KRAS dependency varies in KRAS mutant cancer cell lines. a KRAS mutation status of cell lines used in this study. NSCLC, non-small cell lung cancer; PDAC, pancreatic ductal adenocarcinoma b KRAS mutant cell viability upon KRAS depletion. Cells were treated with KRAS siRNAs (siKRAS \#1, \#2) for $96 \mathrm{~h}$. Cell viability was measured by crystal violet staining assay (upper panel). siRNA interference efficiency was measured by immunoblotting analysis (lower panel). The error bars represent the mean \pm SD of four replicates. ${ }^{*} P<0.05 ;{ }^{* *} P<0.01 ;{ }^{* * *} P<0.001$, n.s., not significant. c, d MAPK and AKT signaling changes upon KRAS depletion in KRAS-dependent (c) and KRAS-independent (d) cells. Cells were treated with KRAS siRNAs (siKRAS \#1, \#2) for $48 \mathrm{~h}$ before being subjected to immunoblotting analysis. e Cell sensitivity to ERK inhibitors. Cells were treated with the ERK inhibitor BVD-523 for $72 \mathrm{~h}$, and cell viability was measured by SRB assay. The error bars represent the mean \pm SD of triplicates. NC, scrambled siRNA used as a negative control

AsPC-1, and Panc 10.05 cells, showed a moderate effect in response to KRAS depletion, while the remaining cells barely responded and were considered KRAS-independent cells (Fig. 1b). The knockdown efficiency was comparable among the tested cells.

KRAS activation is known to drive oncogenic malignancy, mainly via two downstream pathways, the RAF/MEK/ERK pathway, also known as the MAPK/ERK pathway, and the phosphoinositide 3-kinase (PI3K) pathway [6]. We hence examined the key signaling molecules in these two downstream pathways. In both KRAS-dependent and KRAS-independent cancer cells, KRAS depletion led to the universal downregulation of the MAPK/ERK pathway, as indicated by the decreased phosphorylation levels of c-RAF and ERK, while the impact on the PI3K/AKT pathway was variable among the cell lines (Fig. 1c, d; S1a). Consistently, pharmacological inhibition of the MAPK/ERK pathway using the clinically tested ERK kinase inhibitor BVD-523 resulted in substantial cell growth inhibition in KRAS-dependent A549 and $\mathrm{H} 358$ cells, which was in great contrast to KRAS-independent cell lines, such as H441 and H2030 (Fig. 1e; S1b). These results identified a subset of KRAS mutant cancer cells whose growth was highly dependent on KRAS activation and established a linkage between KRAS oncogenic activation and MAPK/ERK signaling activation instead of the PI3K/AKT pathway.

PDE $\delta$ depletion is not necessarily associated with cell growth inhibition in KRAS-dependent cells

We then attempted to assess whether the disruption of PDE $\delta$ in KRAS-dependent cells could lead to growth inhibition. As the selectivity of the reported PDE $\delta$ inhibitors remains in question $[13,16]$, we chose to use two independent siRNAs to specifically knockdown PDE6D, which encodes PDES, and cell growth was then assessed using a crystal violet staining assay. In nine KRASdependent or partially dependent cells, only a small fraction of the 

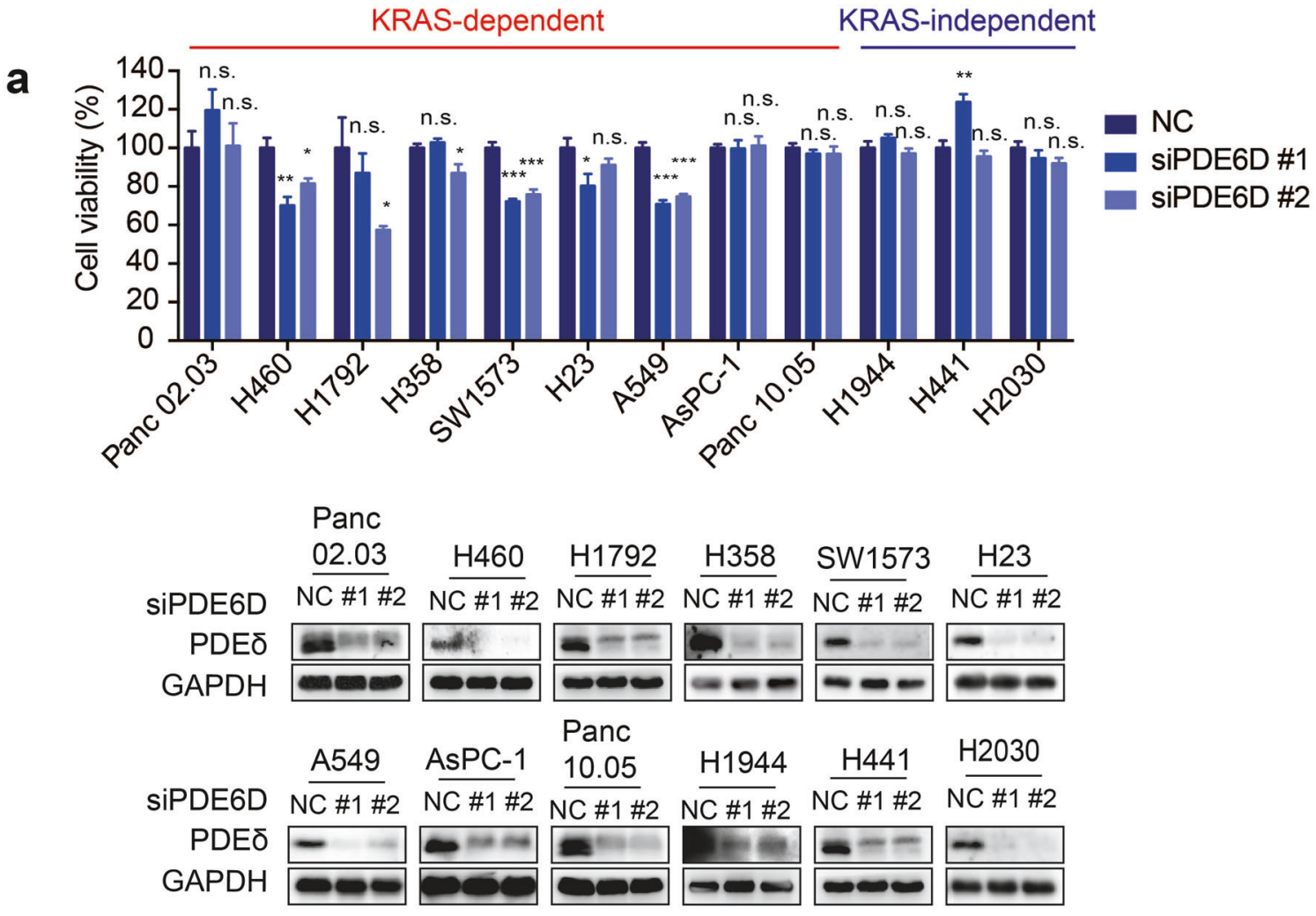

b

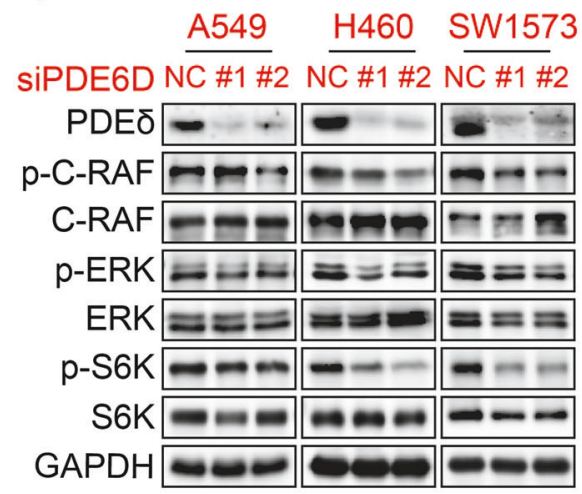

C

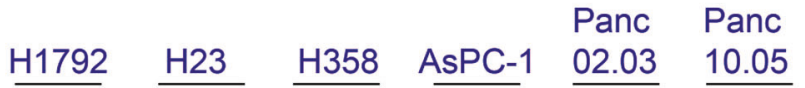

SiPDE6D NC \#1 \#2 NC\#1 \#2 NC \#1 \#2 NC \#1 \#2 NC \#1 \#2 NC \#1 \#2

\begin{tabular}{|c|c|c|c|c|c|c|}
\hline PDEס & 6 & - & $-\cdots$ & $0 \quad-1$ & $\Leftrightarrow-20$ & $9 \mathrm{~m}$ \\
\hline $\mathrm{p}-\mathrm{C}-\mathrm{RAF}$ & $\sigma \mathrm{m}=$ & $\cdots-$ & +10 & $2 \pi$ & Eacas & $\Leftrightarrow \oplus$ \\
\hline C-RAF & --- & 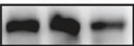 & $-m$ & $-\infty$ & 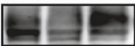 & $-m=$ \\
\hline$p-$ & $z=2$ & $\Rightarrow==$ & 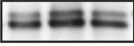 & $=-=$ & $==-$ & $=\equiv$ \\
\hline ERK & 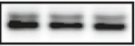 & $=3 \equiv$ & $= \pm=$ & $=8 \varepsilon$ & $==2$ & $\equiv \equiv \equiv$ \\
\hline $6 \mathrm{~K}$ & 26 & $-\infty-\infty$ & $-\infty$ & $-\pi$ & man & --- \\
\hline S & $-\infty-\infty$ & -50 & $=0$ & & $=-2$ & \\
\hline - - מחס & & & $=6$ & & $\rightarrow \rightarrow \infty$ & $5=0$ \\
\hline
\end{tabular}

Fig. 2 MAPK/ERK signaling alteration is associated with the response to PDE $\delta$ inhibition in KRAS-dependent cancer cells. a KRAS mutant cell viability upon PDE $\delta$ depletion. KRAS-dependent cells were treated with PDE $\delta$ siRNAs (siPDE6D \#1, \#2) for 96 h. Cell viability was measured by crystal violet staining assay (upper panel). siRNA interference efficiency was measured by immunoblotting (lower panel). The error bars represent the mean \pm SD of four replicates. ${ }^{*} P<0.05 ;{ }^{*} P<0.01 ;{ }^{* *} P<0.001$, n.s., not significant. $\mathbf{b}$, $\mathbf{c}$ MAPK and AKT signaling changes upon PDES depletion in PDES-dependent (b) and PDES-independent cells (c). Cells were treated with siRNAs (siPDE6D \#1, \#2) for 48 h before being subjected to immunoblotting analysis. NC, scrambled siRNA used as a negative control

cell lines, namely, A549, H460, and SW1573 cells, responded partially to PDES, as indicated by partially suppressed growth inhibition (Fig. 2a, upper panel); however, PDE $\delta$ was similarly depleted in all the tested cells (Fig. 2a, lower panel). In KRAS-independent cells, as expected, none of the cells responded to PDES depletion (Fig. 2a).

We asked whether the differential cell response upon PDE $\delta$ knockdown could possibly result from the differential impact on MAPK/ERK signaling. By simultaneously analyzing the MAPK/ERK pathway in PDE $\delta$-responsive (red) and nonresponsive cells (blue), we discovered that siRNA depletion of PDE $\delta$ effectively diminished $\mathrm{p}-\mathrm{S} 6 \mathrm{~K}$, a surrogate marker for PDE $\delta$ function $[12,13,16]$.
However, MAPK/ERK signaling was only decreased in PDESresponsive cells (Fig. 2b). For example, in A549, H460, and SW1573 cells, phosphorylation levels of c-RAF and ERK were effectively decreased. In contrast, in the rest of the cells in which PDE $\delta$ knockdown barely affected cell growth (Fig. 2c), alterations to RAF/MEK/ERK signaling were not observed. These results suggested that cell growth inhibition was associated with diminished MAPK/ERK signaling.

Together, these results suggest that PDE $\delta$ depletion is not necessarily associated with cell growth inhibition in KRASdependent cells. Effective MAPK/ERK signaling blockage is required for the growth inhibition. 
a

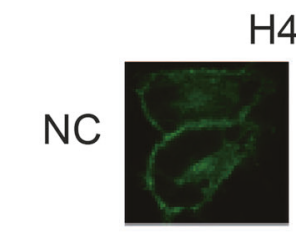

$\mathrm{H} 460$
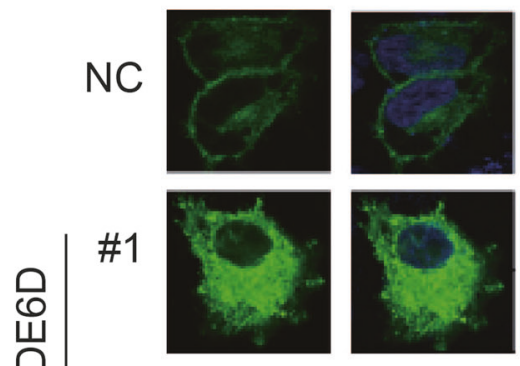

\#2
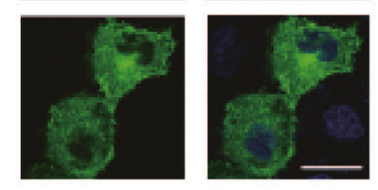

b

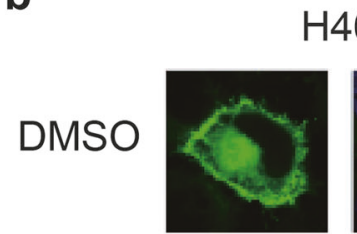

$\mathrm{H} 460$
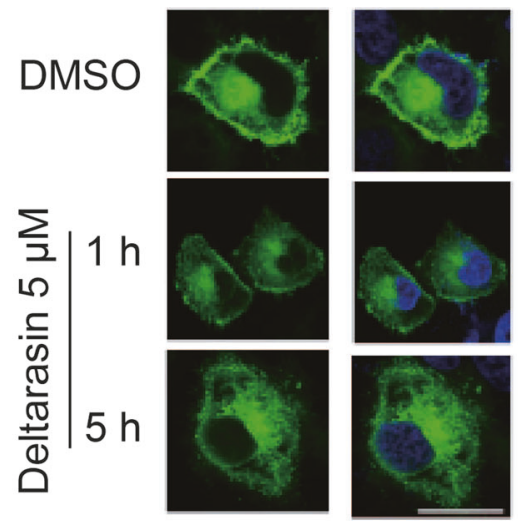

C

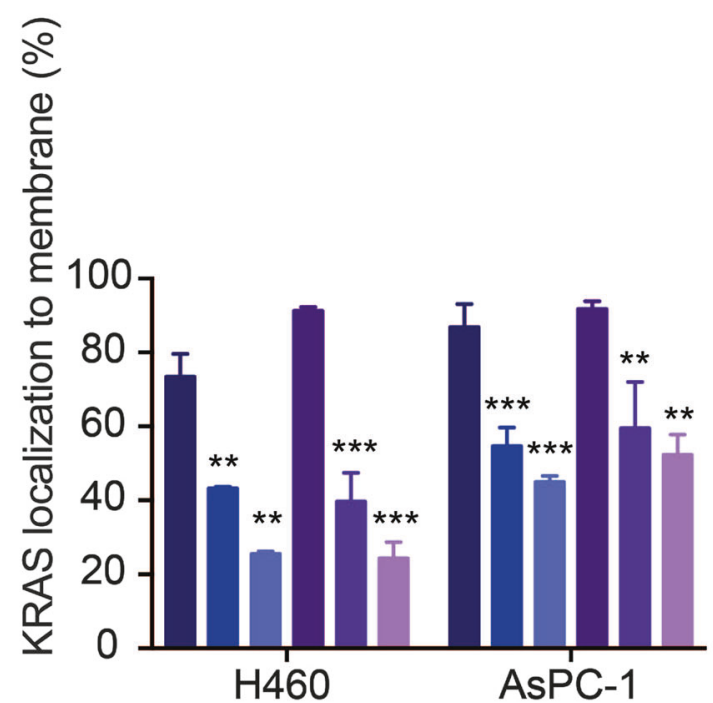

AsPC-1

NC
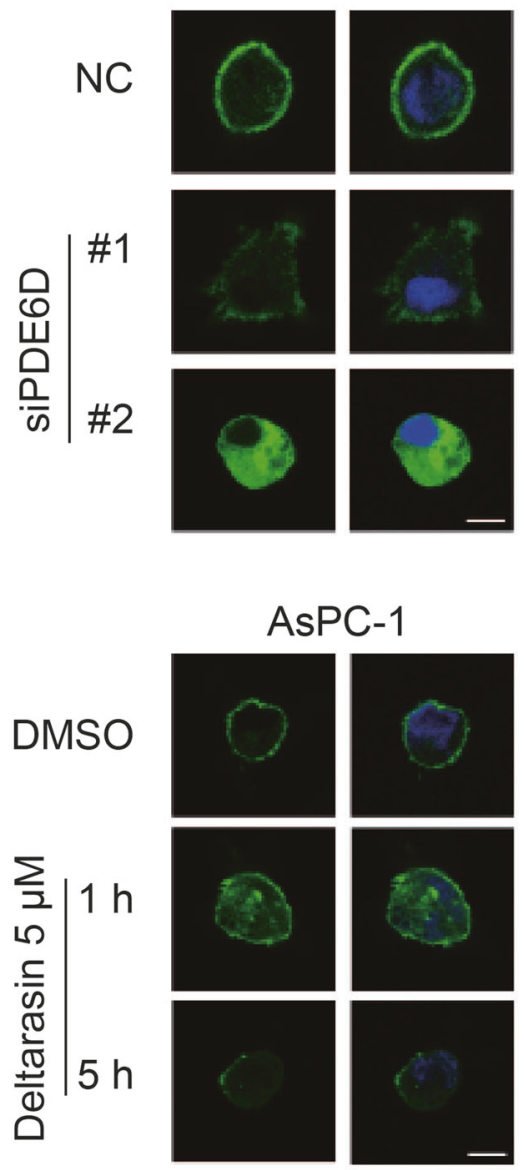
a

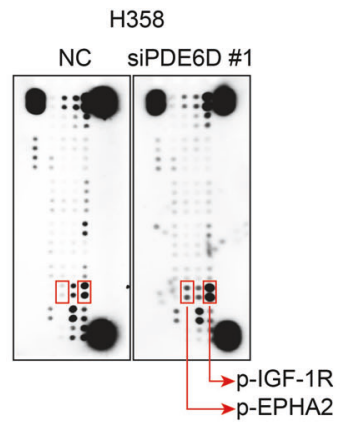

b

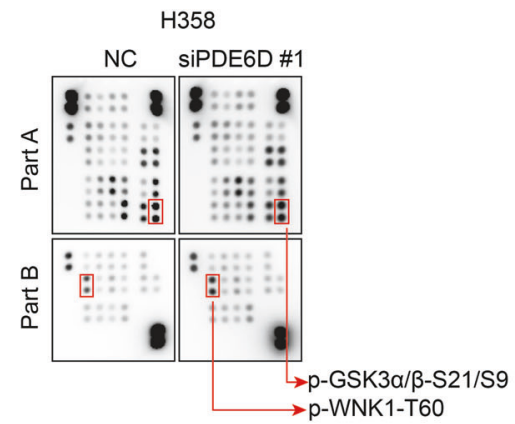

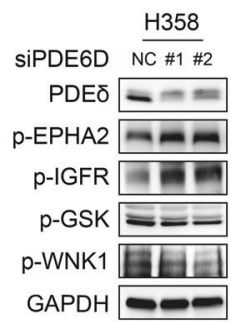

d

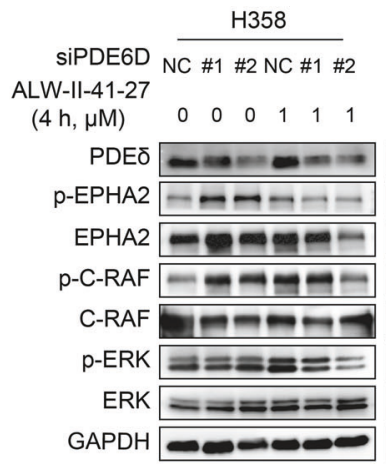

e

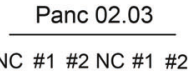

$\begin{array}{llllll}0 & 0 & 0 & 1 & 1 & 1\end{array}$

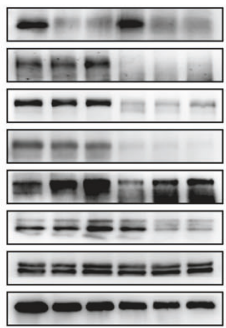
SiPDE6D NC \#1 \#2 NC \#1 \#2 NC \#1 \#2 NC \#1 \#2 BMS-754807 (5 h, $\mu \mathrm{M})$

\begin{tabular}{|c|c|c|}
\hline PDEס & 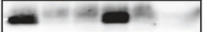 & $-\quad-$ \\
\hline GF-IR & 르를 & mpranger \\
\hline IGF-IR & - & 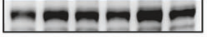 \\
\hline -C-RAF & $=\infty$ & 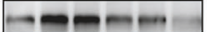 \\
\hline C-RAF & 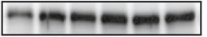 & 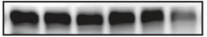 \\
\hline p-ERK & $\equiv \equiv \equiv \equiv \equiv$ & -Eニ=ニ- \\
\hline ERK & $=a=-=$ & =ニニニニニ \\
\hline -AKT & $\operatorname{men-1}$ & $-\infty \boldsymbol{n}$ \\
\hline AKT & mancones & $=0 \pi 00$ \\
\hline & $-\infty$ & - \\
\hline
\end{tabular}

f

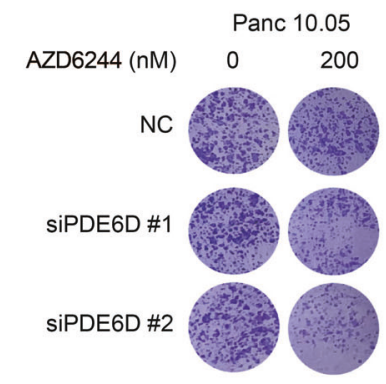

g
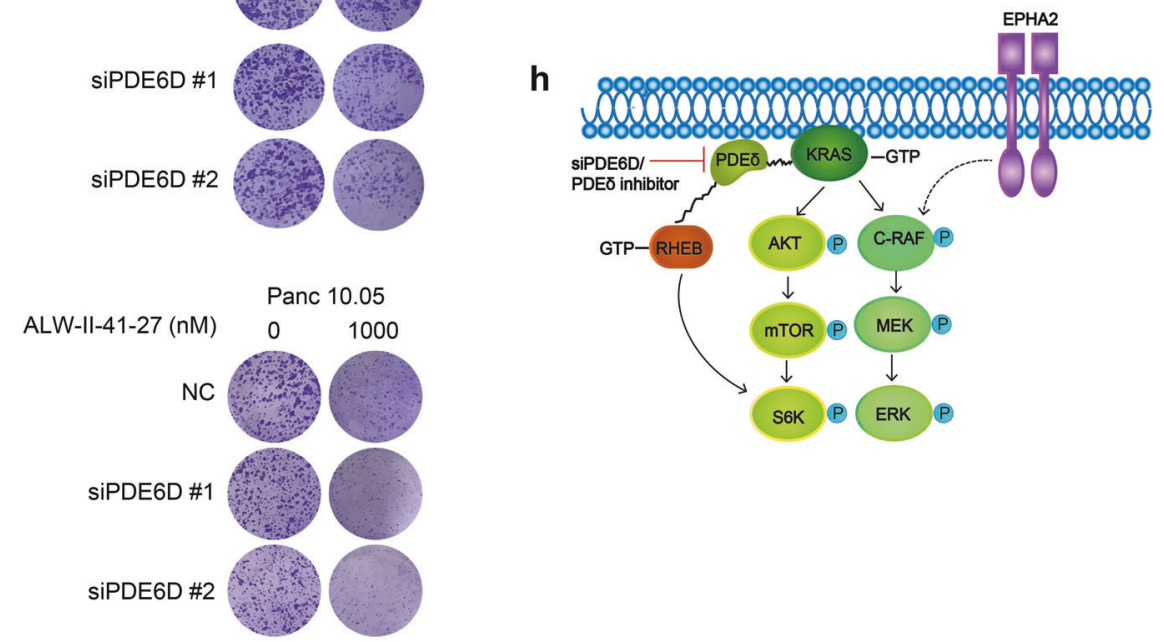

Fig. 4 Feedback activation of EPHA2 results in compensatory MAPK activation in PDE $\delta$-depleted cells. a, b Phospho-RTK antibody and phospho-kinase antibody arrays. Cells were treated with siPDE6D (\#1) for $48 \mathrm{~h}$, and the cell lysates were subjected to phospho-RTK antibody array (a) or phospho-kinase antibody array (b). c Immunoblotting analysis confirmed the feedback activation of EPHA2 and IGF1R in PDESdepleted cells. H358 cells were treated with the indicated siRNA for $48 \mathrm{~h}$ before being subjected to immunoblotting. $\mathbf{d}$ MAPK signaling upon concurrent inhibition of PDES and EPHA2. Cells were transfected with the indicated siRNAs for $48 \mathrm{~h}$ before exposure to the EPHA2 inhibitor ALW-II-41-27 for $4 \mathrm{~h}$. e MAPK signaling upon concurrent inhibition of PDE $\delta$ and IGF1R. Cells were transfected with the indicated siRNAs for 48

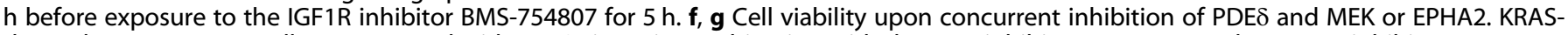
dependent Panc 10.05 cells were treated with PDE $\delta$ siRNA in combination with the MEK inhibitor AZD6244 or the EPHA2 inhibitor ALW-II-4127 for $96 \mathrm{~h}$. Cell viability was measured by crystal violet staining assay. NC, scrambled siRNA used as a negative control. $\mathbf{h}$ A schematic model

GFP-conjugated KRAS was transfected into the cells to visualize KRAS subcellular localization using fluorescence microscopy. In H460 cells, which are sensitive to PDES deficiency, knockdown of PDE6D gene expression efficiently impaired KRAS membrane association (Fig. 3a, left panel). Similar results were obtained using deltarasin, the first reported PDE $\delta$ inhibitor that has been shown to effectively disrupt KRAS plasma membrane localization [13] (Fig. 3b, left panel). 
The assay was also conducted in AsPC-1 cells, in which PDE6D siRNA failed to inhibit cell growth or disrupt MAPK/ERK signaling. Interestingly, in PDE $\delta$-nonresponsive cells, KRAS membrane localization was similarly impaired (Fig. 3a-c). These results indicated that KRAS plasma membrane association was impaired upon PDE $\delta$ depletion in PDE $\delta$-nonresponsive cells, suggesting that the sustained MAPK/ERK pathway activation could possibly result from compensatory upstream signaling.

Feedback activation of EPHA2 activates the MAPK/ERK pathway upon PDE $\delta$ disruption

To explore the possible involvement of compensatory pathways, we used a phospho-RTK antibody and phospho-kinase antibody arrays, which covered 49 phosphorylated human receptor tyrosine kinases (RTKs) and 43 intracellular kinase phosphorylation sites, respectively. This approach allowed us to screen the kinases that were activated upon PDE $\delta$ disruption. At $48 \mathrm{~h}$ following PDE $\delta$ knockdown, we observed the feedback activation of several protein kinases, including IGF1R, EPHA2, GSK3a/ $\beta$, and WNK-1, in H358 cells, which were shown to be nonresponsive to PDE $\delta$ knockdown (Fig. $4 a, b$ ). Among these altered kinases, the upregulation of IGF1R and EPHA2 induced by siPDE6D treatment was confirmed in an independent immunoblotting analysis (Fig. 4c).

We next tested whether the feedback activation of IGF1R and EPHA was responsible for persistent MAPK/ERK activation upon PDE6D knockdown. To this end, cells were treated simultaneously with IGF1R or EPHA2 inhibitors [17, 18] and PDE6D siRNA. MAPK/ ERK signaling was examined using an immunoblotting analysis. It was observed that the EPHA2 inhibitor ALW-II-41-27 could diminish the MAPK/ERK pathway signaling in a panel of PDEסnonresponsive cell lines, including H358, Panc 02.03, Panc 10.05, and AsPC- 1 cells, in which PDE6D siRNA alone failed to reduce KRAS-induced MAPK/ERK signaling (Fig. 4d; S2a). In contrast, treatment with the IGF1R inhibitor BMS-754807 barely affected the phosphorylation of c-RAF and ERK upon PDE $\delta$ knockdown (Fig. 4e; S2b).

These results suggested that the feedback activation of EPHA2 activated the MAPK/ERK pathway upon PDE $\delta$ disruption. We further applied the EPHA2 inhibitor ALW-II-41-27 or EPHA2 siRNA to test whether the cells could be re-sensitized to PDE $\delta$ inhibition. Indeed, we discovered that while PDE $\delta$ depletion alone barely affected cell viability, combined treatment with ALW-II-41-27 (Fig. 4g) and EPHA2 siRNA (Fig. S2c) largely inhibited the growth of Panc 10.05 cells. Similar results were obtained by combining a MEK inhibitor and PDE6D siRNA (Fig. 4f).

\section{DISCUSSION}

Targeting KRAS plasma membrane association has been recently proposed for exploration as a new therapeutic opportunity for KRAS mutant cancer, which highlights PDE $\delta$ as an attractive target to impede KRAS oncogenic signaling. However, the current understanding of this new strategy is very limited, especially considering that the complex signaling network of PDE $\delta$ is known to be involved. In addition, given the heterogeneous properties of KRAS mutant cancer, it is conceivable that PDE $\delta$ inhibition may not yield therapeutic benefits in broad KRAS mutant contexts. It will be important to stratify the subset responsive to PDE $\delta$ inhibition. In this study, we discovered that only a fraction of KRAS-dependent cells responds to PDE $\delta$ inhibition, although KRAS plasma membrane association is efficiently impaired. The feedback activation of EPHA2 accounts for the limited response to PDE $\delta$ inhibition via a mechanism that involves the compensatory activation of the MAPK/ERK signaling (Fig. 4h). These insights help gain a better understanding of the PDE $\delta$-targeted therapeutic strategy and suggest the combined inhibition of EPHA2 and PDE $\delta$ as a potential therapy for KRAS mutant cancer.
It remains unclear how EPHA2 is specifically activated upon PDE $\delta$ inhibition in KRAS-dependent cancer. Currently, there is no evidence suggesting a direct link between PDE $\delta$ and EPHA2. Limited evidence thus far may suggest the involvement of CDC42, which possibly mediates the association between PDE $\delta$ and EPHA2. EPHA2 is known to regulate RHO/RAC/CDC42 $[19,20]$. It has also been shown that PDE $\delta$ could interact with CDC42 [14]. One possibility is that the impairment of PDE $\delta$ could induce the feedback response of CDC42, which further provokes EPHA2 via a feedback loop. This hypothesis will be interesting to explore in the future.

In addition, a large number of studies have shown that EPHA2 interacts with KRAS and downstream signaling pathways [21] EPHA2 belongs to receptor tyrosine kinase families that regulate multiple downstream signaling pathways, including the MAPK/ERK and PI3K/ AKT/mTOR pathways $[19,22,23]$. It is possible that impaired PDE $\delta$ causes KRAS to lose control over its direct downstream MAPK/ERK signaling pathway. In this scenario, a higher level of EPHA2 or its active status is more likely to allow the feedback activation of the MAPK/ERK pathway. Indeed, we compared the EPHA2 expression in KRAS-dependent cells using Cell Line Encyclopedia (CCLE) data sets and found that EPHA2 expression was relatively higher in cells that were nonresponsive to PDE $\delta$ inhibition than in those that were responsive to PDE $\delta$ inhibition (Fig. S3a). In parallel, the expression level of IGF-IR did not differ between PDES-responsive and nonresponsive cell lines (Fig. S3b). We further examined the phosphorylation level of EPHA2 in these cells. EPHA2 was often activated, as indicated by the basal EPHA2 phosphorylation level, in PDES-nonresponsive cells (Fig. S3c). Certainly, the two aforementioned possibilities could exist in parallel. A more in-depth study will be required to carefully dissect how EPHA2 is specifically provoked upon PDE $\delta$ depletion.

Finally, in this study, we discovered that EPHA2-induced MAPK/ ERK signaling is essential for determining KRAS mutant cancer survival. Due to the complexity of signaling pathways involving KRAS and the heterogeneous properties of KRAS mutants, it remains unclear whether other pathways beyond EPHA2-MAPK signaling are involved in determining the survival of KRAS mutant cancer. It is possible that the novel strategy of combining PDE6D and EPHA2 inhibition discovered in this study may be applicable to only a subset of KRAS mutant cancer.

\section{ACKNOWLEDGEMENTS}

This work was supported by the National Natural Science Foundation of China (No. 81821005), and the Strategic Priority Research Program of the Chinese Academy of Sciences (Nos XDA12020345 and XDA12020230).

\section{AUTHOR CONTRIBUTIONS}

MH conceived the whole project; $\mathrm{BX}$ and MH initiated the project; MYG and JD provided supervision; YHC performed the research and analyzed the data; $\mathrm{HL}$ performed the experiments in the initial stage of the project; XMW, NS, and ST provided technique assistance; $\mathrm{MH}$ and $\mathrm{YHC}$ wrote the paper. All authors approved the final version of the paper.

\section{ADDITIONAL INFORMATION}

The online version of this article (https://doi.org/10.1038/s41401-019-0268-y) contains supplementary material, which is available to authorized users.

Competing interests: The authors declare no competing interests.

\section{REFERENCES}

1. Cox AD, Der CJ. Ras history: the saga continues. Small GTPases. 2010;1:2-27.

2. Cox AD, Fesik SW, Kimmelman AC, Luo J, Der CJ. Drugging the undruggable RAS: mission possible? Nat Rev Drug Discov. 2014;13:828-51.

3. Papke B, Der CJ. Drugging RAS: know the enemy. Science. 2017;355:1158-63. 
4. Karnoub AE, Weinberg RA. Ras oncogenes: split personalities. Nat Rev Mol Cell Biol. 2008;9:517-31.

5. Fehrenbacher N, Bar-Sagi D, Philips M. Ras/MAPK signaling from endomembranes. Mol Oncol. 2009;3:297-307.

6. Hubbard PA, Moody CL, Murali R. Allosteric modulation of Ras and the PI3K/AKT/ mTOR pathway: emerging therapeutic opportunities. Front Physiol. 2014;5:478.

7. Martini M, De Santis MC, Braccini L, Gulluni F, Hirsch E. PI3K/AKT signaling pathway and cancer: an updated review. Ann Med. 2014;46:372-83.

8. Pylayeva-Gupta Y, Grabocka E, Bar-Sagi D. RAS oncogenes: weaving a tumorigenic web. Nat Rev Cancer. 2011;11:761-74.

9. Thompson H. US National Cancer Institute's new Ras project targets an old foe. Nat Med. 2013;19:949-50.

10. Mansi L, Viel E, Curtit E, Medioni J, Le Tourneau C. Targeting the RAS signalling pathway in cancer. Bull Cancer. 2011;98:1019-28.

11. Baehr W. Membrane protein transport in photoreceptors: the function of PDEdelta: the Proctor lecture. Invest Ophthalmol Vis Sci. 2014;55:8653-66.

12. Chandra A, Grecco HE, Pisupati V, Perera D, Cassidy L, Skoulidis F, et al. The GDIlike solubilizing factor PDEdelta sustains the spatial organization and signalling of Ras family proteins. Nat Cell Biol. 2011;14:148-58.

13. Zimmermann G, Papke B, Ismail S, Vartak N, Chandra A, Hoffmann M, et al. Small molecule inhibition of the KRAS-PDEdelta interaction impairs oncogenic KRAS signalling. Nature. 2013;497:638-42.

14. Kuchler $P$, Zimmermann $G$, Winzker $M$, Janning $P$, Waldmann $H$, Ziegler $S$. Identification of novel PDEdelta interacting proteins. Bioorg Med Chem. 2018;26:1426-34.
15. Singh A, Greninger P, Rhodes D, Koopman L, Violette S, Bardeesy N, et al. A gene expression signature associated with "K-Ras addiction" reveals regulators of EMT and tumor cell survival. Cancer Cell. 2009;15:489-500.

16. Papke B, Murarka S, Vogel HA, Martin-Gago $P$, Kovacevic $M$, Truxius DC, et al. Identification of pyrazolopyridazinones as PDEdelta inhibitors. Nat Commun. 2016;7:11360.

17. Bid HK, London CA, Gao J, Zhong H, Hollingsworth RE, Fernandez S, et al. Dual targeting of the type 1 insulin-like growth factor receptor and its ligands as an effective antiangiogenic strategy. Clin Cancer Res. 2013;19:2984-94.

18. Duxbury MS, Ito H, Zinner MJ, Ashley SW, Whang EE. EphA2: a determinant of malignant cellular behavior and a potential therapeutic target in pancreatic adenocarcinoma. Oncogene. 2004;23:1448-56.

19. Pasquale EB. Eph-ephrin bidirectional signaling in physiology and disease. Cell. 2008;133:38-52.

20. Fang WB, Ireton RC, Zhuang G, Takahashi T, Reynolds A, Chen J. Overexpression of EPHA2 receptor destabilizes adherens junctions via a RhoA-dependent mechanism. J Cell Sci. 2008;121:358-68.

21. Zhou Y, Sakurai $H$. Emerging and diverse functions of the EphA2 noncanonical pathway in cancer progression. Biol Pharm Bull. 2017;40:1616-24.

22. Amato KR, Wang S, Hastings AK, Youngblood VM, Santapuram PR, Chen H, et al Genetic and pharmacologic inhibition of EPHA2 promotes apoptosis in NSCLC. J Clin Invest. 2014;124:2037-49.

23. Amato KR, Wang S, Tan L, Hastings AK, Song W, Lovly CM, et al. EPHA2 blockade overcomes acquired resistance to EGFR kinase inhibitors in lung cancer. Cancer Res. 2016;76:305-18. 